\title{
TECNOLOGIAS DE INFORMAÇÃO E COMUNICAÇÃO COMO RECURSO FACILITADOR NO PROCESSO DE APRENDIZAGEM
}

\author{
Beatriz Féba de Sales, Renata Portela Rinaldi \\ Universidade Estadual Paulista Julio de Mesquita Filho - UNOESTE, Departamento de Educação, Presidente Prudente, \\ SP. E-mail: beatrizfeba8@gmail.com
}

\begin{abstract}
RESUMO
Este trabalho visa apresentar parte da pesquisa realizada para o Trabalho de Conclusão de Curso de Licenciatura em Pedagogia da FCT/UNESP, que tem por objetivo investigar o uso das Tecnologias de Informação e Comunicação - TIC como recurso facilitador no processo de aprendizagem das crianças de anos iniciais do ensino fundamental. De abordagem qualitativa, desenvolveu-se via pesquisa bibliográfica e os dados foram coletados em três bases de dados, sendo: ANPED (Associação Nacional de Pós-graduação e Pesquisa em Educação) no GT 16 Educação-Comunicação; SIED-EnPED (Simpósio Internacional de Educação a Distância e Encontro de Pesquisadores em Educação a Distância) no período de 2012 e 2014; base de dados SciELO, no período de 2005 e 2017. O aporte teórico foi extraído de livros, artigos, documentos monográficos e periódicos que tratam dessa temática. Os resultados apontam que é possível utilizar as TIC para facilitar o processo de ensino-aprendizagem de crianças no processo inicial de escolarização.

Palavras-chave: Tecnologias de informação e comunicação, Recursos pedagógicos digitais, Aprendizagem.

\section{INFORMATION AND COMMUNICATION TECHNOLOGIES AS A RESOURCE FACILITATOR IN THE LEARNING PROCESS}

\begin{abstract}
This work aims to present part of the research realized for the Course Completion Work of the Pedagogy of FCT/UNESP, which aims to investigate the use of the Information and Communication Technologies (ICT) as a facilitator resource in the process of learning children from early years of elementary school. From a qualitative approach, it was developed through bibliographic research and the data were collected in three databases, being: ANPED (National Association of Postgraduate and Research in Education) in GT 16 Education-Communication; SIED-EnPED (International Symposium on Distance Education and Meeting of Researchers in Distance Education) in the period of 2012 and 2014; SciELO database, in the period of 2005 and 2017. The theoretical contribution was extracted from books, articles, monographic documents and periodicals that work with this theme. The results indicate that it is possible to use ICT to facilitate the teaching-learning process of children in the initial schooling process.
\end{abstract}

Keywords: Information and communication technologies, Digital pedagogical resources, Learning. 


\title{
INTRODUÇÃO
}

No presente texto, apresentamos os resultados parciais de uma pesquisa de Conclusão de Curso intitulada "Tecnologias de informação e comunicação como recurso facilitador no processo de aprendizagem" que teve início em junho de 2017.

A pesquisa é decorrente de uma questão intrigante que se deu a partir de observações realizadas em nosso cotidiano. Verificamos, por meio de diversas mídias que as crianças já nos seus primeiros meses de vida estão tendo contato com os novos aparelhos digitais e os adultos, em geral, não conseguem ficar sem eles.

Nesse sentido, torna-se necessário primeiramente, definir o significado de Tecnologia de Informação e Comunicação (TIC). De acordo com Oliveira; Moura e Souza (2015, p. 3),

\begin{abstract}
As Tecnologias da Informação e Comunicação referidas como TIC são consideradas como sinônimo das tecnologias da informação (TI). Contudo, é um termo geral que frisa o papel da comunicação na moderna tecnologia da informação. Entende-se que TIC consistem de todos os meios técnicos usados para tratar a informação e auxiliar na comunicação. Em outras palavras, TIC consistem em TI, bem como quaisquer formas de transmissão de informações e correspondem a todas as tecnologias que interferem e mediam os processos informacionais e comunicativos dos seres. Ainda, podem ser entendidas como um conjunto de recursos tecnológicos integrados entre si, que proporcionam por meio das funções de software e telecomunicações, a automação e comunicação dos processos de negócios, da pesquisa científica e de ensino e aprendizagem.
\end{abstract}

Segundo os autores estudados para a realização da pesquisa, as TIC são muito utilizadas atualmente nas escolas, sejam elas públicas ou privadas. Em geral, as crianças ao menos uma vez na semana possuem aulas no laboratório de informática onde têm acesso ao computador, à internet, às redes sociais como Facebook entre outros. No entanto, ainda é frequente que vários professores não utilizam os recursos tecnológicos no processo de ensino, isso decorre por motivos variados que vão desde a falta de conhecimento para o uso pedagógico do recurso até a própria falta do recurso nas escolas.

Pode-se dizer que, entre outros aspectos, o que está faltando para os professores aderirem em suas aulas os artefatos tecnológicos e assim tornar a forma de ensinar mais prazerosa para as crianças é o investimento contínuo na formação desses professores para o uso pedagógico desses recursos.

Para tanto, neste texto, apresentaremos alguns resultados do estudo realizado até o momento, buscando apresentar experiências de uso de TIC no ensino com crianças, por exemplo, jogos ou artefatos digitais para facilitar o processo de ensino aprendizado das crianças que possuem dificuldades e também das crianças que não possuem nenhum tipo de dificuldade para aprender.

Acreditamos que é um estudo relevante para o campo de educação, pois permite compreender novos caminhos para aprendizagem da docência.

Desta forma, propomos a pesquisa de Conclusão de Curso com o objetivo de analisar quais os recursos de Tecnologia de Informação e Comunicação podem auxiliar as crianças no momento do seu aprendizado, para que assim ele se torne um momento agradável, atrativo e fácil.

\section{METODOLOGIA}

Para a realização da pesquisa bibliográfica, iniciamos pelo levantamento bibliográfico nas seguintes bases de dados: Associação Nacional de Pós-Graduação e Pesquisa em Educação ${ }^{1}$ (ANPED), especificamente o Grupo de Trabalho (GT) 16 - Educação e Comunicação; Simpósio Internacional de Educação a Distância e Encontro de Pesquisadores em Educação a Distância ${ }^{2}$

\footnotetext{
${ }^{1}$ Disponível no endereço: <http://www.35reuniao.anped.org.br/trabalhos/123-gt16>

${ }^{2}$ Disponível no endereço: <http://sistemas3.sead.ufscar.br/ojs/index.php/sied/issue/view/1> e <http://www.sied-

enped2014.ead.ufscar.br/ojs/index.php/2014>
} 
(SIED-EnPED) e Base de dados SciELO³. Foram definidas as seguintes palavras-chave para padronizar as buscas nesta etapa: TIC, tecnologias digitais, jogos e tecnologias, TIC como recurso de aprendizagem.

O recorte temporal para a pesquisa dos trabalhos na base de dados da ANPED e do SIEDEnPED foi de 2012 a 2014, a opção por trabalhar com as publicações desse biênio ocorreu porque os eventos ocorrem bianualmente e são importantes eventos que discutem a temática de artefatos digitais na educação e $\mathrm{EaD}$, assim como julgamos um período suficiente para a contextualização da temática para um trabalho de conclusão de curso. Para as buscas na base de dados SciELO ampliamos o período de tempo para 2005-2017.

Após a seleção dos textos, por meio do levantamento bibliográfico, o processo de análise se caracteriza como de natureza descritivo e analítica. Ou seja, realizamos inicialmente a leitura na íntegra dos materiais, posteriormente nova leitura e fichamento. Por fim, foi realizada a análise a partir da identificação de conceitos trabalhados pelos autores, metodologia utilizada e identificação dos principais resultados. Após esse processo, retomamos o objetivo da pesquisa e passamos a escrever a análise sobre o objeto investigado.

\section{RESULTADOS}

Quadro 1. Artigos selecionados por descritores na base de dados do Scielo.

\begin{tabular}{|l|l|l|}
\hline Descritores & $\begin{array}{l}\text { Quantidade de artigos } \\
\text { encontrados }\end{array}$ & $\begin{array}{l}\text { Quantidade de artigos } \\
\text { selecionados }\end{array}$ \\
\hline TICS & 3 & 3 \\
\hline Tecnologias Digitais & 5 & 5 \\
\hline Jogos e tecnologias recurso de & 1 & 1 \\
\hline $\begin{array}{l}\text { TIC como } \\
\text { aprendizagem }\end{array}$ & 10 & 1 \\
\hline TOTAL: & 10 & 10 \\
\hline
\end{tabular}

Fonte: Sistematizado pelas autoras a partir dos resultados da pesquisa.

Como podemos notar no quadro 1 acima, foram selecionados poucos textos com os descritores elencados. Selecionamos, assim, 10 artigos sendo: 3 com o descritor TIC (BALADELI; BARROS; ALTOE, 2012; BONILLA e PRETTO, 2007; COUTO, 2014;). Um total de 5 textos com o descritor tecnologias digitais foram selecionados (ALMEIDA, 2008; LUCENA, 2016; MONTEIRO e OSÒRIO, 2015; OLIVEIRA; MOURA; SOUZA, 2015; PRETTO, 2011;). Um texto com o descritor jogos e tecnologias (LIMBERGER; MÜLLER; PINTO, 2016) e um com o descritor TIC como recurso de aprendizagem no ensino (NAGUMO e TELES, 2016).

Quadro 2. Artigos selecionados na base dedados da ANPED - GT 16

\begin{tabular}{|l|l|l|l|}
\hline Grupo de Trabalhos & $\begin{array}{l}\text { Total de } \\
\text { Artigos }\end{array}$ & $\begin{array}{l}\text { Total de Artigos } \\
\text { Descartados }\end{array}$ & $\begin{array}{l}\text { Total de Artigos } \\
\text { Selecionados }\end{array}$ \\
\hline GT16 - Educação e Comunicação & 14 & 7 & 7 \\
\hline
\end{tabular}

Fonte: Sistematizado pelas autoras a partir dos resultados da pesquisa.

Dos trabalhos selecionados, observamos as seguintes características complementares aos descritores definidos inicialmente: um trabalho com o descritor ambientes virtuais e aprendizagem (VARGAS NETTO, 2012); um com os descritores crianças e redes sociais (MACEDO, 2012); um com os descritores culturas digitais e mobilidade (FERREIRA, 2012); um trabalho com os descritores materiais didáticos digitais, rede e aprendizagem (RODRIGUES e MESQUITA, 2012); um

${ }^{3}$ Disponível no endereço: <http//www.scielo.org/php/index.php 
trabalho com o descritor cotidiano na cibercultura (SANTOS e SANTOS, 2012); um com os descritores identidades infantis, club penguin e animalamina (SARAIVA e KIRCHOF, 2012); e um trabalho com o descritor TIC (LOUREIRO e LOPES, 2012).

Quadro 3. Artigos selecionados na base de dados do SIED-EnPED 2012

\begin{tabular}{|l|l|l|l|}
\hline Grupo de trabalhos & $\begin{array}{l}\text { Total de } \\
\text { Artigos }\end{array}$ & $\begin{array}{l}\text { Total de Artigos } \\
\text { Descartados }\end{array}$ & $\begin{array}{l}\text { Total de Artigos } \\
\text { Selecionados }\end{array}$ \\
\hline SIED 2012- Comunicação Oral & 112 & 110 & 2 \\
\hline
\end{tabular}

Fonte: Sistematizado pelas autoras a partir dos resultados da pesquisa.

Os dois trabalhos selecionados para análise foram encontrados a partir dos seguintes descritores: um com o descritor ambiente virtual e aprendizagem (CÂMARA e JOÃO, 2012) e um trabalho com os descritores games, virtualidade, ludicidade, imaginário e educação infantil (JUNIOR e KISHIMOTO, 2012). Por último, nos trabalhos publicados no SIED-EnPED 2014 foram encontrados 138 trabalhos dentro do evento. Mas, foram descartados 135 trabalhos que não encontravam dentro do tema pesquisado, sendo selecionado apenas 3 trabalhos que se encontravam no tema que buscamos. Nesta etapa, os descritores utilizados também foram diferenciados devido ao evento, mas também se enquadram dentro das palavras-chave que nos baseamos em nossas pesquisas que são: tecnologias de informação e comunicação, recursos pedagógicos digitais, aprendizagem, e para chegar a esses novos descritores olhamos palavras chaves de trabalho por trabalho. Dessa maneira, utilizamos os seguintes descritores: alfabetização, educação, informática e software, tecnologias digitais, audiovisuais, web conferência e videoconferência.

Quadro 4. Artigos selecionados na base de dados do SIED-EnPED 2014

\begin{tabular}{|l|l|l|l|}
\hline Grupo de trabalhos & Total de Artigos & $\begin{array}{l}\text { Total de Artigos } \\
\text { Descartados }\end{array}$ & $\begin{array}{l}\text { Total de Artigos } \\
\text { Selecionados }\end{array}$ \\
\hline $\begin{array}{l}\text { SIED 2014- } \\
\text { Comunicação Oral }\end{array}$ & 138 & 135 & 3 \\
\hline
\end{tabular}

Fonte: Sistematizado pelas autoras a partir dos resultados da pesquisa.

Dos três trabalhos selecionados foram encontrados os seguintes descritores: um trabalho com os descritores alfabetização, educação, informática e software (ALVES e CASTRO, 2014), um com os descritores tecnologias digitais, audiovisuais, webconferência e videoconferência (ARRUDA e DUTRA, 2014) e um trabalho com os descritores dispositivos digitais com internet, smartphones e tablets, metacognição, percepção de gênero, interação social (ROCHA e MILL, 2014).

\section{DISCUSSÃO}

O enfoque dos artigos encontrados nas três bases de dados: SciELO, ANPED - GT16, SIEDEnPED 2012 e 2014 é de nos apresentar novos meios tecnológicos que poderão ser utilizados no processo ensino-aprendizagem das crianças, por exemplo: tecnologias digitais como computadores, tablets e meios tecnológicos da informática.

\section{CONCLUSÃO}

No decorrer da pesquisa, observamos que algumas das fontes pesquisadas indicam que não há estudos que demonstram qual a melhor forma tecnológica a ser utilizada para auxiliar no processo ensino-aprendizagem das crianças. Ou seja, encontramos referências que nos traz vários exemplos de uso de tecnologias, mas nenhum que nos relacione explicitamente o melhor meio tecnológico para o ensino de crianças nos anos iniciais. Por outro lado, os autores encontrados 
que debatem o uso de tecnologias para o ensino aprendizado, em sua maioria, relacionam o tema com outras formas de se ensinar as crianças e não especificamente através dos meios tecnológicos.

\section{REFERÊNCIAS}

ALMEIDA, M. E. B. Tecnologias na Educação: dos caminhos trilhados aos atuais desafios. Bolema: Boletim de Educação Matemática, Rio Claro, v. 21, n.29, p. 99-129, 2008. Disponível em: <http://www.redalyc.org/articulo.oa?id=291221870006>. Acesso em: 05 ago. 2018.

ALVES, F. A. M.; CASTRO, A. B. B. de. O uso de softwares como recurso didático: na alfabetização infantil: Uma proposta. In: Simpósio Internacional de Educação a Distância: Encontro de Pesquisadores em Educação a Distância (SIED:EnPED), 2014, São Carlos. Anais eletrônicos. Disponível em: <https://www.siedenped2014.ead.ufscar.br/ojs/index/php/2014/article/view/567/285>. Acesso em: 05 ago. 2018.

ARRUDA, D. E. P.; DUTRA, C. S. O uso de tecnologias audiovisuais como mediadoras no contexto educacional: vídeo aulas, vídeo conferência e web conferências. In: Simpósio Internacional de Educação a Distância: Encontro de Pesquisadores em Educação a Distância (SIED:EnPED), 2014, São Carlos. Anais eletrônicos. Disponível em: <http://www.siedenped2014.ead.ufscar.br/ojs/index.php/2014/article/view/720/203>. Acesso em: 05 ago. 2018.

BALADELI, A. P. D.; BARROS, M. S. F.; ALTOE, A. Desafios para o professor na sociedade da informação. Educar em Revista, Curitiba, n. 45, p. 155-165, set. 2012. Disponível em: $<$ http://www.scielo.br/scielo.php?script=sci_arttext\&pid=S0104-

$40602012000300011 \&$ Ing=en\&nrm=iso>. Acesso em 05 ago. 2018.

BONILLA, M. H. S.; PRETTO, N. De L. Formação de professores: as TIC estruturando dinâmicas curriculares horizontais. In: ARAÚJO, B.; FREITAS, K. S. de. Educação a distância no contexto brasileiro: experiências em formação inicial e formação continuada. Salvador: ISP/UFBA, 2007. p. 73-92. Disponível em: <http://www.proged.ufba.br/ead/EADlivro2.pdf>. Acesso em: 05 ago. 2018.

CÂMARA, J. M.; JOÃO, H. A. Proposta de uso do ambiente virtual de aprendizagem Fronter como estratégia de facilitação para o processo de ensino-aprendizagem. In: Simpósio Internacional de Educação a Distância: Encontro de Pesquisadores em Educação a Distância (SIED:EnPED), 2012, São Carlos. Anais eletrônicos. Disponível em: <http://sistemas3.sead.ufscar.br/ojs1/index.php/sied/article/view/198/95>. Acesso em: 05 ago. 2018.

COUTO, H. H. O. de M. Jovens professores no contexto da prática e as tecnologias de informação e comunicação (TIC). Educação \& Sociedade, Campinas, v. 35, n. 126, p. 257-272, mar. 2014. Disponível em: <http://www.scielo.br/scielo.php?script=sci_arttext\&pid=S010173302014000100015\&lng=en\&nrm=iso>. Acesso em: 05 ago. 2018.

FERREIRA, H. M. C. Cultura da mobilidade: Como ela aparece na escola? 35a Reunião Anual da Anped, 2012, Porto de Galinhas, Ipojuca-PE. Anais eletrônicos. Disponível em: <http://35reuniao.anped.org.br/images/stories/trabalhos/GT16\%20Trabalhos/GT161756_int.pdf>. Acesso em: 05 ago. 2018. 
JUNIOR, W. A.; KISHIMOTO, T. M. Uso de games por crianças: virtualidade e simulação no espaço lúdico. In: Simpósio Internacional de Educação a Distância: Encontro de Pesquisadores em Educação a Distância (SIED:EnPED), 2012, São Carlos. Anais eletrônicos. Disponível em: <http://sistemas3.sead.ufscar.br/ojs1/index.php/sied/article/view/274>. Acesso em: 05 ago. 2018.

LIMBERGER, Leticia Staub; MÜLlER, César Augusto; PINTO, Maira Meira. Aprendizagens e videogames. Revista Ibero-Americana de Estudos em Educação, v.1, n.3, p. 1149-1168, jul.-set. 2016. Disponível em: <https://periodicos.fclar.unesp.br/iberoamericana/article/view/7852/5902>. Acesso em: 05 ago. 2018.

LOUREIRO, C. B.; LOPES, M. C. Tecnologias da informação e comunicação: Outras formas de condução das condutas. 35a Reunião Anual da Anped, 2012, Porto de Galinhas, Ipojuca-PE. Anais eletrônicos. Disponível

em: <http://35reuniao.anped.org.br/images/stories/trabalhos/GT16\%20Trabalhos/GT162284_int.pdf>. Acesso em: 05 ago. 2018.

LUCENA, S. Culturas digitais e tecnologias móveis na educação. Educar em Revista., Curitiba, n. 59, p. 277-290, mar. 2016.2 Disponível em: <http://www.scielo.br/scielo.php?script=sci_arttext\&pid=S0104$40602016000100277 \&$ Ing=pt\&nrm=iso>. Acesso em 05 ago. 2018.

MACEDO, N. M. R. Crianças e redes sociais: Uma proposta de pesquisa online. 35a Reunião Anual da Anped, 2012, Porto de Galinhas, Ipojuca-PE. Anais eletrônicos. Disponível em: <http://35reuniao.anped.org.br/images/stories/trabalhos/GT16\%20Trabalhos/GT162101_int.pdf>. Acesso em: 05 ago. 2018.

MASETTO, M. T. Mediação pedagógica e o uso das tecnologias. In: MORAN, J. M.; MASETTO, M. T.; BEHRENS, M. A. Novas tecnologias e mediação pedagógica. 10 ed. Campinas, SP: Papirus, 2006, p.133-173.

MONTEIRO, A. F.; OSÒRIO, A. J. Novas tecnologias, riscos e oportunidades na perspetiva das crianças. Revista Portuguesa de Educação, Braga , v. 28, n. 1, p. 35-57, jun. 2015. Disponível em: $<$ http://www.scielo.mec.pt/scielo.php?script=sci_arttext\&pid=S087191872015000100003\&lng=es\&nrm=iso>. Acesso em: 05 ago. 2018. DOI: 10.21814/rpe.7049. https://doi.org/10.21814/rpe.7049

NAGUMO, E.; TELES, L. F. O uso do celular por estudantes na escola: motivos e desdobramentos. Revista Brasileira de Estudos Pedagógicos, Brasília, v. 97, n. 246, p. 356-371, ago. 2016. Disponível em: $<$ http://www.scielo.br/scielo.php?script=sci_arttext\&pid=S217666812016000200356\&lng=en\&nrm=iso>. Acesso em: 05 ago. 2018.

OLIVEIRA, C. de; MOURA, S. P.; SOUZA, E. R. Tic's na Educação: A utilização das tecnologias da informação e comunicação na aprendizagem do aluno. Pedagogia em Ação, Belo Horizonte, v. 7, n. 1, p. 75-95, $2015 . \quad$ Disponível em: <http://periodicos.pucminas.br/index.php/pedagogiacao/article/view/11019/8864>. Acesso em: 05 ago. 2018.

PRETTO, N. De L. O desafio de educar na era digital: educações. Revista Portuguesa de Educação, Braga, v. 24, n. 1, p. 95-118, 2011. Disponível em: 
$<$ http://www.scielo.mec.pt/scielo.php?script=sci_arttext\&pid=S0871-

91872011000100005\&lng=pt\&nrm=iso>. Acesso em: 05 ago. 2018.

ROCHA, E. G.; MILL, D. Smartphones e tablets como mediadores para interação social, desenvolvimento cognitivo e percepção de gênero na educação. In: Simpósio Internacional de Educação a Distância: Encontro de Pesquisadores em Educação a Distância (SIED:EnPED), 2014, São Carlos. Anais eletrônicos. Disponível em: <http://www.siedenped2014.ead.ufscar.br/ojs/index.php/2014/article/view/838>. Acesso em: 05 ago. 2018.

RODRIGUES, E. S. J.; MESQUITA, O. A. de. Materiais didáticos digitai em rede e aprendizagem: Reflexões críticas. 35a Reunião Anual da Anped, 2012, Porto de Galinhas, Ipojuca-PE. Anais eletrônicos. Disponível <http://35reuniao.anped.org.br/images/stories/trabalhos/GT16\%20Trabalhos/GT162311_int.pdf>. Acesso em: 05 ago. 2018.

SANTOS, R. dos; SANTOS, E. O. dos. Pesquisando nos cotidianos da cibercultura: Uma experiência de pesquisa-formação multirreferencial. 35a Reunião Anual da Anped, 2012, Porto de Galinhas, Ipojuca-PE. Anais eletrônicos. Disponível em: <http://35reuniao.anped.org.br/images/stories/trabalhos/GT16\%20Trabalhos/GT162195_int.pdf>. Acesso em: 05 ago. 2018.

SARAIVA, K. S.; KIRCHOF, E. R. Produção de identidades infantis nos sites club penguin e animalamina. 35a Reunião Anual da Anped, 2012, Porto de Galinhas, Ipojuca-PE. Anais eletrônicos. Disponível em: <http://35reuniao.anped.org.br/images/stories/trabalhos/GT16\%20Trabalhos/GT161604_int.pdf>. Acesso em: 05 ago. 2018.

VARGAS NETTO, M. J. As práticas de espectatura com o Youtube como campo de aprendizado e pesquisa. 35a Reunião Anual da Anped, 2012, Porto de Galinhas, Ipojuca-PE. Anais eletrônicos. Disponível em: <http://35reuniao.anped.org.br/images/stories/trabalhos/GT16\%20Trabalhos/GT161740_int.pdf>.

VELOSO, M. M. S. de A. O professor e a autoria no contexto da cibercultura: redes da criação no cotidiano da escola. 2014. 279 f. Tese (Doutorado em Educação) - Programa de Pós-Graduação em Educação, Faculdade de Educação, Universidade Federal da Bahia, Salvador. Disponível em: <https://repositorio.ufba.br/ri/bitstream/ri/15664/1/TESE\%20MARISTELA\%20MIDLEJ\%20VELOSO. pdf>. Acesso em: 05 ago. 2018. 